\title{
Effect of ryanodine on sinus node recovery time determined in vitro
}

J.W.M. Bassani,

C.M.G. Godoy and

R.A. Bassani
Centro de Engenharia Biomédica and Departamento de Engenharia Biomédica,

Faculdade de Engenharia Elétrica e de Computação,

Universidade Estadual de Campinas, Campinas, SP, Brasil

\section{Correspondence}

J.W.M. Bassani

Departamento de Engenharia

Biomédica

FEEC, UNICAMP

Caixa Postal 6040

13083-970 Campinas, SP

Brasil

Fax: + 55-19-289-3346

E-mail: bassani@deb.fee.unicamp.br

Research partially supported by CNPq (Nos. 300069/95-2 and

301905/84-3-NV). Publication supported by FAPESP. The present address of C.M.G. Godoy is Núcleo de Pesquisas Tecnológicas, Universidade de Mogi das Cruzes, 08780-210 M ogi das Cruzes, SP, Brasil.

Received September 9, 1998 Accepted April 20, 1999

\section{Abstract}

Evidence has indicated that the sarcoplasmic reticulum (SR) might be involved in the generation of spontaneous electrical activity in atrial pacemaker cells. We report the effect of disabling the SR with ryanodine $(0.1 \mu \mathrm{M})$ on the sinus node recovery time (SNRT) measured in isolated right atria from 4-6-month-old male Wistar rats. Electrogram and isometric force were recorded at $36.5^{\circ} \mathrm{C}$. Two methods for sinus node resetting were used: a) pulse: a single stimulus pulse interpolated at coupling intervals of 50,65 or $80 \%$ of the regular spontaneous cycle length (RCL), and b) train: a 2-min train of pulses at intervals of 50,65 or $80 \%$ of RCL. Corrected SNRT (cSNRT) was calculated as the difference between SNRT (first spontaneous cycle length after stimulation interruption) and RCL. Ryanodine only slightly increased RCL $(<10 \%)$, but decreased developed force by $90 \%$. When the pulse method was used, cSNRT $(\sim 40 \mathrm{~ms})$, which represents intranodal/atrial conduction time, was independent of the coupling interval and unaffected by ryanodine. However, cSNRT obtained by the train method was significantly higher for shorter intervals between pulses, indicating the occurrence of overdrive suppression. In this case, ryanodine prolonged cSNRT in a rate-dependent fashion, with a greater effect at shorter intervals. These results indicate that: a) a functional SR, albeit important for force development, does not seem to play a major role in atrial automaticity in the rat; b) disruption of cell $\mathrm{Ca}^{2+}$ homeostasis by inhibition of SR function does not appear to affect conduction; however, it enhances overdrive-induced depression of sinusal automaticity.

The sinus node is considered to be the primary pacemaker responsible for heart automaticity. Disturbances in sinusal automaticity itself, in its chronotropic response to autonomic modulators and/or in conduction of the electrical impulse through the right atrium may lead to rhythm dysfunctions generally referred to as sick sinus syndrome (1). Programmed electrical stimulation of the

\section{Key words}

- Sinus node recovery time

- O verdrive suppression

- Electrical stimulation

- Calcium

- Sarcoplasmic reticulum

- Ryanodine heart and estimation of the sinus node recovery time (SNRT) have been used as a clinical approach to evaluate atrial automaticity/conduction (e.g., 2,3).

In general terms, SNRT is the interval required for resumption of spontaneous, regular electrical activity after pacemaker resetting by electrical stimulation. Two methods have been used to estimate this variable. One 
is based on the interpolation of a single, premature electrical stimulus between two spontaneous depolarizations to achieve sinus resetting (4). If the previous regular spontaneous cycle length (RCL) is subtracted from the interval between stimulus delivery and the first spontaneous depolarization (i.e., SNRT), the corrected SNRT (cSNRT) is obtained. cSNRT thus represents the sum of the anterograde and retrograde conduction times between the site of stimulation and the pacemaker, plus the intranodal conduction time $(4,5)$. The second method $(2)$, which is technically simpler, involves pacing the atrium at a rate higher than the spontaneous frequency, in order to entrain the sinus node. However, it has been shown that this method may yield comparatively higher cSNRT values when pacing is done at relatively higher rates (see e.g., 5,6). This additional delay for the spontaneous rhythm to resume is termed overdrive suppression.

$\mathrm{Ca}^{2+}$ has been implicated in impulse conduction in cardiac tissue (7). The sarcoplasmic reticulum (SR) is the main $\mathrm{Ca}^{2+}$ store in mammalian cardiac myocytes, representing most of the contraction-activating $\mathrm{Ca}^{2+}$ pool during excitation (8). There have been indications that the SR might be involved in the generation of spontaneous activity in atrial pacemaker cells. The electrogenic extrusion, via $\mathrm{Na}^{+} / \mathrm{Ca}^{2+}$ exchange, of $\mathrm{Ca}^{2+}$ released from the SR may generate an inward current which appears to play an important role in diastolic depolarization of atrial latent pacemaker cells (9). It has been shown that irreversible inhibition of SR function markedly depresses spontaneous activity in these cells $(9,10)$.

In the present study, we investigated the role of a functional SR in the determination of the clinically used parameter cSNRT, using a previously characterized in vitro approach (6). In these experiments, the plant alkaloid ryanodine was used to disrupt SR function by impairing SR $\mathrm{Ca}^{2+}$ accumulation.

Right atria were isolated from adult male
Wistar rats (4-6 months old) and incubated in Krebs-Henseleit solution of the following composition: $122.3 \mathrm{mM} \mathrm{NaCl}, 4.6 \mathrm{mM} \mathrm{KCl}$, $2.5 \mathrm{mM} \mathrm{CaCl}_{2}, 1.2 \mathrm{mM} \mathrm{KH}_{2} \mathrm{PO}_{4}, 17.7 \mathrm{mM}$ $\mathrm{NaHCO}_{3}, 1.2 \mathrm{mM} \mathrm{MgSO} 4,11.1 \mathrm{mM}$ glucose, $\mathrm{pH} 7.4$, saturated with $95 \% \mathrm{O}_{2} / 5 \%$ $\mathrm{CO}_{2}$, at $36.5^{\circ} \mathrm{C}$. The upper extremity of the atrial appendage was connected to a force transducer (model F-60, Narco Bio-Systems, Austin, TX, USA) and the lower one was impaled on a tetrapolar platinum electrode used for electrogram detection and stimulation (6). Electrical stimulation and signal acquisition were controlled by a computerbased system (11). Electrogram and isometric tension signals were acquired via a customized acquisition system (analog/digital converter CAD1236; acquisition software Aqdados 4, Lynx Tech. Electr. Ltd., São Paulo, SP, Brazil). After application of $0.5 \mathrm{gf}$ resting tension, an equilibration period of 45 min was allowed to elapse.

After a stable RCL was reached, SNRT was determined by application of : a) a 2-min long train of pulses at intervals of 50, 65 or $80 \%$ of RCL (train method, see Figure 1A), and $\mathrm{b}$ ) a single, premature stimulus (5-ms duration, $1.2 \mathrm{x}$ threshold current) after an interval corresponding to 50,65 or $80 \%$ of RCL (pulse method, see Figure 1B), as described by Marques et al. (6). These intervals (pulses) or frequencies (trains) were randomly applied. After SNRT was obtained with both methods under control conditions, $0.1 \mu \mathrm{M}$ ryanodine (Calbiochem, La Jolla, CA, USA) was added to the bath solution. After 20-min incubation, when the full effects of ryanodine were attained, the stimulation protocol was repeated. SNRT values obtained from the electrogram were considered acceptable only when they indicated sinus node resetting. cSNRT was calculated subtracting the previous mean RCL from SNRT.

Data obtained from 5 preparations were compared by two-way analysis of variance. Statistically significant differences were con- 
sidered to occur for values of $\mathrm{P}<0.05$.

Ryanodine showed a dramatic negative inotropic effect (Figure 1C), reducing peak twitch force by $90 \%(\mathrm{P}<0.01)$, which is in agreement with previous observations in rat myocardium (8). The negative chronotropic effect, however, was small and nonsignificant, with an increase of less than $8 \%$ in RCL (Figure 1C).

As shown in Figure 2A, cSNRT values obtained by the pulse method under control conditions were similar regardless of pulse prematurity $(45 \pm 10,42 \pm 10$ and $41 \pm 10 \mathrm{~ms}$ for intervals of 50,65 and $80 \%$ RCL, respectively). Ryanodine did not significantly change cSNRT $(\mathrm{P}>0.9)$ under these conditions $(31 \pm 3,37 \pm 6$ and $35 \pm 6 \mathrm{~ms}$ for intervals of 50,65 and $80 \%$ RCL, respectively), suggesting that atrial/internodal conduction times do not seem to be affected by loss of SR function.

However, when the train method was used, analysis of variance indicated a significant influence $(\mathrm{P}<0.05)$ of pulse interval on cSNRT. As shown in Figure 2B, in control experiments a negative relationship between cSNRT and interval was observed, so that cSNRT at the shortest interval $(64 \pm 10 \mathrm{~ms}$ at $50 \%$ of RCL) was twice as long as that recorded with the interval closer to RCL (33 $\pm 4 \mathrm{~ms}$ at $80 \%$ of RCL). The latter was not significantly different from the values obtained with the pulse method. This indicates that rapid pacing introduced a component apparently due to depression of sinus automaticity (overdrive suppression). SR inhibition with ryanodine caused an interval-dependent prolongation of cSNRT $(\mathrm{P}<0.05)$ : while no effect was observed at $80 \%$ of RCL ( $34 \pm 4 \mathrm{~ms})$, cSNRT was markedly increased after ryanodine at $50 \%$ of RCL $(624 \pm 187$ $\mathrm{ms})$. This result indicates potentiation of overdrive suppression by impairment of SR function.

Ryanodine greatly suppresses contractility in heart muscle. At nanomolar concentrations it binds to high-affinity sites in the SR
$\mathrm{Ca}^{2+}$ release channels, locking them open in a subconductance state. This promotes continuous leakage of the stored $\mathrm{Ca}^{2+}$ and disrupts normal excitation-contraction coupling, which normally involves activation of SR $\mathrm{Ca}^{2+}$ release by the $\mathrm{Ca}^{2+}$ pool that enters the cell via sarcolemmal $\mathrm{Ca}^{2+}$ channels during the action potential (for a review, see Ref. 8).

Sinus node cells are also able to store considerable amounts of $\mathrm{Ca}^{2+}$ in the SR (10). However, the role of the SR in the automaticity of these cells is still unclear. Li et al. (12) have described a strong negative chronotropic effect of ryanodine in rabbit sinus node cells. However, SR disabling with ryanodine or thapsigargin has shown minimal effects on the duration of spontaneous cycle length in cat sinus cells, while the automaticity of atrial latent pacemaker cells and $\mathrm{Ca}^{2+}$ overloaded myocytes was greatly suppressed

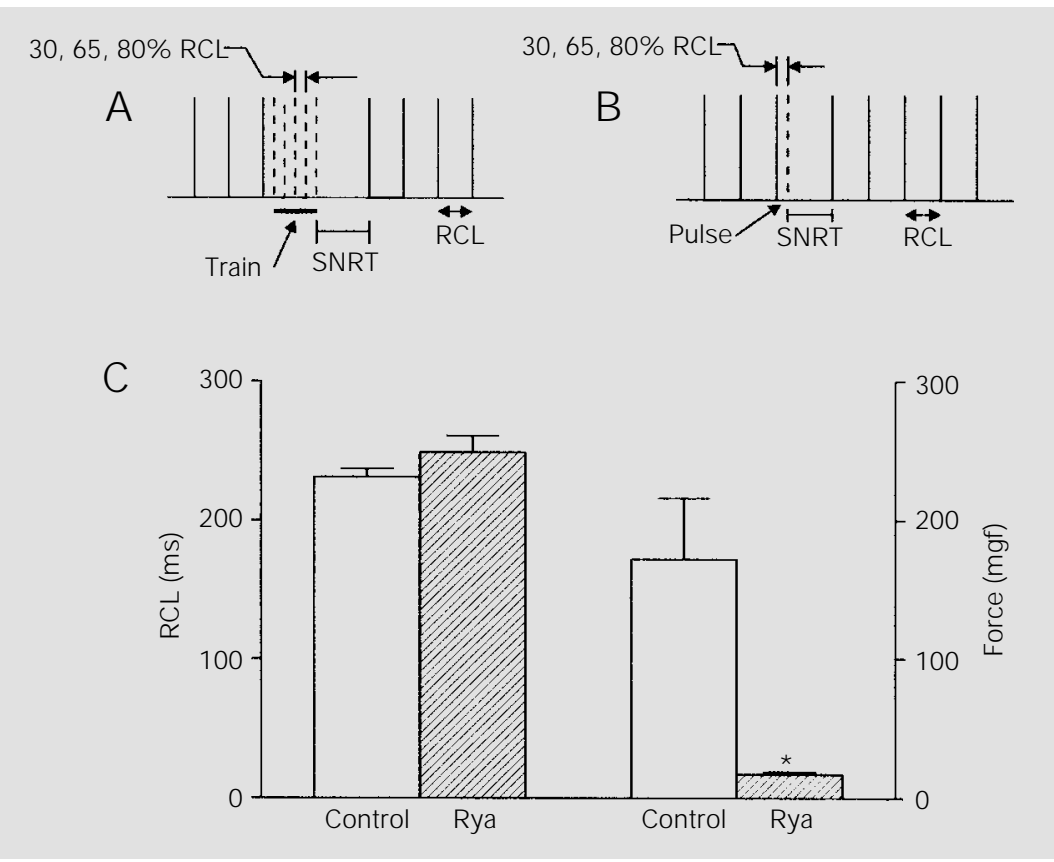

Figure 1 - Stimulation protocols for the determination of sinus node recovery time (SNRT) in isolated rat right atria by the train method (A) and the pulse method (B). Stimuli are represented as broken lines, whereas solid lines represent spontaneous beats at steadystate ( $\mathrm{RCL}$ represents the spontaneous, regular cycle length). Panel C shows the chronotropic and inotropic effects of $0.1 \mu \mathrm{M}$ ryanodine (Rya) on isolated rat right atria. Bars on the left indicate mean \pm SEM RCL, while bars on the right indicate mean \pm SEM isometric active tension. Data from 5 preparations are reported. $* \mathrm{P}<0.01$ compared to control (analysis of variance). 
Figure 2 - Corrected sinus node recovery times (CSNRT) obtained for isolated rat right atria before (control) and after $0.1 \mu \mathrm{M}$ ryanodine application (Rya). Panels A and $B$ present cSNRT values obtained with the pulse and train methods, respectively (see text for details). Means \pm SEM of 5 experiments are reported. by this treatment $(9,10)$. These apparently contradictory results might reflect cell- and species-dependent variation in the importance of the different currents for generation of pacemaker activity. Although electrical activity of individual sinus node cells was not recorded in the present experiments, the small effect of ryanodine on RCL suggests that the SR does not play a major role in the generation of sinus automaticity in the rat.

Conduction time (retrograde plus anterograde) may be estimated as the cSNRT value obtained with the pulse method $(4,5)$. The lack of an effect of ryanodine on this variable also indicates that an important role of the SR in atrial/intranodal conduction of excitation is unlikely.

However, ryanodine greatly enhanced overdrive suppression. Automaticity suppression after rapid pacing has already been described both in vivo (e.g., 2,3,5) and in vitro $(5,6,13,14)$. The mechanisms responsible for this effect in atrial tissue have not yet been ascertained. In addition to pacemaker shift, the main mechanisms proposed

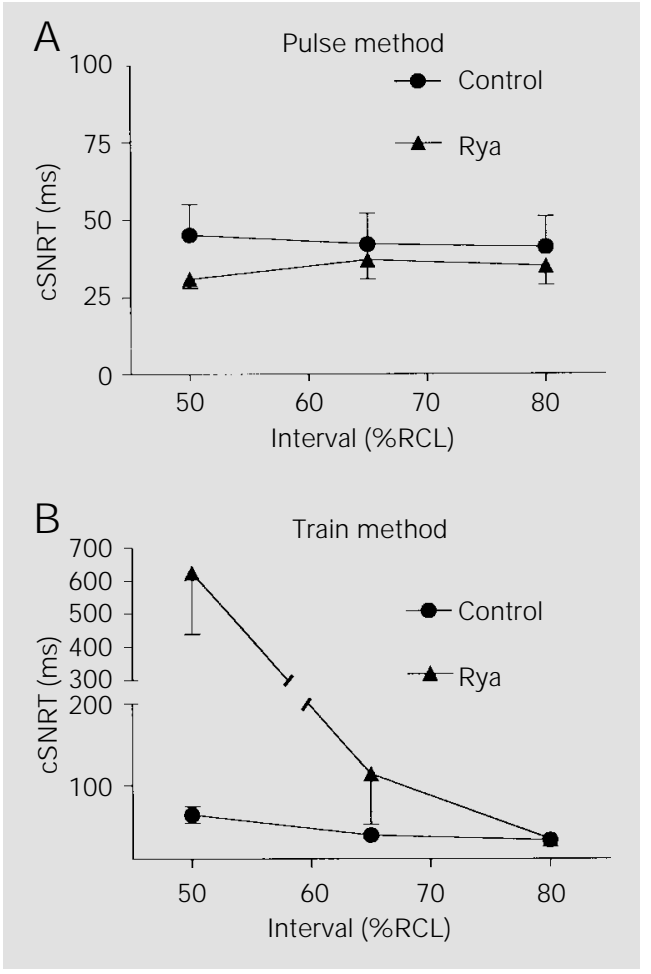

include: a) acetylcholine (ACh) release during pacing (13): although pacing may induce $\mathrm{ACh}$ release in isolated right atria (11) and exogenous ACh enhances overdrive suppression $(5,6,13)$, the observations that overdrive suppression is not abolished by atropine $(5,6)$ and also occurs in isolated sinus node cells (15) suggest that ACh release may not be essential for automaticity suppression by overdrive; b) depression of sinus node automaticity by extracellular $\mathrm{K}^{+}$accumulation due to rapid pacing (13): although this effect may not be ruled out in multicellular preparations, it is unlikely that it alone might account for overdrive suppression in view of the occurrence of suppression in perifused, isolated sinus node cells (15), and also because potentiation of overdrive suppression by increased extracellular $\left[\mathrm{K}^{+}\right]$is mostly due to decreased conduction velocity $(5,14)$; c) membrane hyperpolarization due to activation of the $\mathrm{Na}^{+}-\mathrm{K}^{+}$pump (16): an increase in diastolic membrane potential during overdrive stimulation has been described in Purkinje fibers. However, in isolated sinus node cells, progressive depolarization, rather than hyperpolarization, has been observed during overdrive (15); d) decrease in transmembrane $\mathrm{Ca}^{2+}$ current $\left(\mathrm{I}_{\mathrm{Ca}}\right)$ (15): in sinus node cells, $\mathrm{I}_{\mathrm{Ca}}$ has been considered the major depolarizing current responsible for action potential upstroke (17). In addition to the observation that sarcolemmal $\mathrm{Ca}^{2+}$ channel blockers enhance overdrive suppression $(5,18)$, the existence of a slow $\mathrm{I}_{\mathrm{Ca}}$ inactivation has already been described in single sinus node cells (19). This rate-dependent inactivation of $\mathrm{I}_{\mathrm{Ca}}$ (which is more pronounced at less negative potentials, closer to the diastolic membrane potential of sinus node cells) has been identified as an important mechanism for overdrive suppression in isolated sinus node cells, while other currents also implicated in spontaneous diastolic depolarization $\left(\mathrm{I}_{\mathrm{K}}\right.$ and $\left.\mathrm{I}_{\mathrm{f}}\right)$ do not seem to be involved (15). Our observations, using ryanodine as a tool to disrupt $\mathrm{Ca}^{2+}$ regulation in cardiac 
cells, lend support to this proposal.

The mechanism by which ryanodine potentiates overdrive suppression (without apparent change in impulse conduction time) might involve mainly its ability to prevent effective $\mathrm{Ca}^{2+}$ accumulation by the SR during pacing. Although short-term (up to hours) ryanodine exposure does not affect L- or Ttype $\mathrm{I}_{\mathrm{Ca}}$ in striated muscle (20), ryanodine maintains $\mathrm{SR} \mathrm{Ca}^{2+}$ release channels open in a subconductance state, which leads to constant release of $\mathrm{SR} \mathrm{Ca}^{2+}$. At a higher stimulation frequency, leaked $\mathrm{Ca}^{2+}$ may build up in the cytosol, especially in the subsarcolemmal space, even during diastole. Indeed, a ratedependent, progressive increase in diastolic intracellular $\left[\mathrm{Ca}^{2+}\right]$ has been observed in isolated rabbit myocytes treated with ryanodine (Bassani RA, Bassani JWM and Bers
DM, unpublished results). Subsarcolemmal $\mathrm{Ca}^{2+}$ accumulation might slow down even further $\mathrm{I}_{\mathrm{Ca}}$ recovery from inactivation via the mechanism of $\mathrm{Ca}^{2+}$-dependent inactivation (21), in addition to decreasing current amplitude by reducing the $\mathrm{Ca}^{2+}$ transmembrane electrochemical gradient.

Our results indicate that disturbances of cell $\mathrm{Ca}^{2+}$ homeostasis, which are common in a number of cardiopathic conditions, may enhance automaticity suppression by high rate electrical stimulation, and this should be taken into account when using the SNRT for clinical evaluation of sinus automaticity.

\section{Acknowledgments}

We are grateful to Mr. Gilson B. Maia Jr. for technical assistance.

\section{References}

1. Ferrer MI (1973). The sick sinus syndrome. Circulation, 47: 635-641.

2. Narula OS, Samet P \& J avier RP (1972). Significance of the sinus node recovery time. Circulation, 45: 140-158.

3. Yee R \& Strauss HC (1987). Electrophysiologic mechanisms: sinus node dysfunction. Circulation, 75 (Suppl III): III-12-III-18.

4. Strauss HC, Saroff AL, Bigger Jr JT \& Giardina EGV (1973). Premature atrial stimulation as a key to the understanding of sinoatrial conduction in man. Presentation of data and critical review of the literature. Circulation, 47: 86-93.

5. Prinsze FJ (1993). Cellular aspects of overdrive suppression in the sinoatrial node. Doctoral thesis, University of Amsterdam, The Netherlands.

6. Marques J LB, Bassani RA \& Bassani JWM (1990). Methodology and instrumentation for the in vitro sinus node recovery time determination. J ournal of Pharmacological Methods, 23: 117-127.

7. Sugiura H \& J oyner RW (1992). Action potential conduction between guinea pig ventricular cells can be modulated by calcium current. American J ournal of Physiology, 263: H1591-H1604.

8. Bers DM (1991). Excitation-Contraction Coupling and Cardiac Contractile Force. Kluwer Press, Dordretch, The Netherlands.
9. Lipsius SL, Rubenstein DS \& Zhou Z (1993). Cellular mechanisms of right atrial latent pacemakers. New Trends in Arrhythmias, 19: 11-23.

10. Bassani RA, Bassani J WM, Lipsius SL \& Bers DM (1997). Diastolic SR Ca efflux in atrial pacemaker cells and Ca-overloaded myocytes. American J ournal of Physiology, 273: H886-H892.

11. Bassani J WM, Godoy CMGG \& Bassani RA (1998). Role of acetylcholine in electrically-induced arrhythmia in rat isolated atria. J ournal of Molecular and Cellular Cardiology, 30 (Suppl): A4 (Abstract).

12. Li J , Qu J \& Nathan RD (1997). Ionic basis of ryanodine's negative chronotropic effect on pacemaker cells isolated from the sinoatrial node. American J ournal of Physiology, 273: H2481-H2489.

13. Lange $G$ (1965). Action of driving stimuli from intrinsic and extrinsic sources on in situ cardiac pacemaker tissues. Circulation Research, 17: 449-459.

14. Marques J LB \& Bassani J WM (1989). Influence of the $\mathrm{KCl}$ concentration on rat sinus node recovery time in vitro. Brazilian J ournal of Medical and Biological Research, 22: 803-806.

15. Watanabe E-I, Honjo $H$, Boyett $M R$, Kodama I \& Toyama J (1996). Inactivation of the calcium current is involved in over- drive suppression of rabbit sinoatrial node cells. American J ournal of Physiology, 271: H2097-H2107.

16. Vassale M (1970). Electrogenic suppression of automaticity in sheep and dog Purkinje fibres. Circulation Research, 27: 361-377.

17. Irisawa HH, Brown HF \& Giles W (1993). Cardiac pacemaking in the sinoatrial node. Annual Review of Physiology, 73: 197227.

18. Konishi T (1976). Electrophysiological considerations on sick sinus syndrome. J apanese Circulation J ournal, 40: 194-201.

19. Habushi $Y$, Noda $T$, Nishimura $M \&$ Watanabe $Y$ (1990). Recovery of the inward current from $\mathrm{Ca}^{2+}$-mediated and voltage-dependent inactivation in the rabbit sinoatrial node. J oumal of Molecular and Cellular Cardiology, 22: 469-482.

20. Kenyon J L, McKemy DD, Airey JA \& Sutko J L (1995). Interaction between ryanodine receptor function and sarcolemmal $\mathrm{Ca}^{2+}$ currents. American J ournal of Physiology, 269: C334-C340.

21. Lee KS, Marban E \& Tsien RW (1985). Inactivation of calcium channels in mammalian heart cells: joint dependence on membrane potential and intracellular calcium. J ournal of Physiology, 364: 395411. 